\title{
Transverse Plasma Resonans Mode in an Nonmagnetized Plasma and Its Practical Applications
}

\author{
F. F. Mende ${ }^{*}$ \\ B.I. Verkin Institute for Low Temperature Physics and Engineering, NAS Ukraine, 47 Lenin Ave., Kharkov, Ukraiua \\ *Corresponding author: mende_fedor@mail.ru
}

Received September 28, 2014; Revised October 31, 2014; Accepted November 02, 2014

\begin{abstract}
Is shown that in the nonmagnetized plasma, besides longitudinal Langmuir resonance can exist the transverse plasma resonance. The resonance indicated can exist in the confined plasma. It is known that with the nuclear explosions the electromagnetic radiation in the very wide frequency band is observed, up to the radiofrequency range. And if the emission in field of light range can be explained by the emission of separate atoms, then emission in the region of radio-frequency band can be caused only by collective processes, which occur in the confined plasma. The use of transverse resonance makes it possible to create resonators and band-pass filters, and also lasers on the collective plasma oscillations. Transverse plasma resonance can be used also for the warming-up of plasma and its diagnostics. Is introduced the concept of magnetoelectrokinetic waves.
\end{abstract}

Keywords: plasma, resonance, Langmuir resonance, plasma resonance, cavity, laser

Cite This Article: F. F. Mende, "Transverse Plasma Resonans Mode in an Nonmagnetized Plasma and Its Practical Applications." American Journal of Electrical and Electronic Engineering, vol. 2, no. 5 (2014): 152-158. doi: 10.12691/ajeee-2-5-3.

\section{Introduction}

Until now, was considered that in the nonmagnetized plasma only one Langmuir resonance can exist. The processes, which characterize this resonance, are connected with a longitudinal change in the density of plasma. This resonance cannot emit electromagnetic waves lengthwise. It is known that with the nuclear explosions the electromagnetic radiation in the very wide frequency band is observed, up to the radio-frequency range. And if the emission in field of light range can be explained by the emission of separate atoms, then emission in the region of radio-frequency band can be caused only by collective processes, which occur in the confined plasma.

\section{Electrodynamics of the Nonmagnetized Plasma}

A large quantity of articles in the scientific journals and monographs $[1,2,3,4]$ is devoted to the examination of electrodynamic processes in the plasma. In the publications indicated primary attention is paid to questions of physics of the magnetized plasma, and is only brief description of processes, proceeding in the nonmagnetized plasma. Is described only one Langmuir (plasma) resonance, which was known up to now. It should be noted that with the description of processes in the nonmagnetized plasma in the monographs indicated are terminological and systematic inaccuracies, which must be corrected.
Let us examine the plasmo-like medium, in which be absent the ohmic losses. Such plasma medium is superconductor or the very hot plasma, in which the ohmic losses can be disregarded. In this case the equation of motion of electron takes the form

$$
m \frac{d \vec{V}}{d t}=e \vec{E}
$$

where $\mathrm{m}$ and e is a mass and electron charge, $\vec{E}$ is tension of electric field, $\vec{V}$ is speed of the motion of charge.

Taking into account that current density

$$
\vec{j}=n e \vec{V},
$$

we obtain from Eq. (2.2):

$$
\vec{j}_{L}=\frac{n e^{2}}{m} \int \vec{E} d t
$$

In Eqs. (2.2) and (2.3) the value $n$ determines the charge density. After introducing the designation $[5,6]$

$$
L_{k}=\frac{m}{n e^{2}}
$$

Let us write down

$$
\vec{j}_{L}=\frac{1}{L_{k}} \int \vec{E} d t
$$

In this case the value $L_{k}$ presents the specific kinetic inductance of plasmo-like medium. Its existence connected with the fact that charge, having a mass, possesses inertia properties. In spite of the obviousness of 
this record of the density of inductive current, it was for the first time proposed in the work [7].

Pour on Eq. (2.5) it will be written down for the case of harmonics:

$$
\vec{j}_{L}=-\frac{1}{\omega L_{k}} E_{0} \cos \omega t
$$

From Eqs. (6.5) and (6.6) is evident that $\vec{j}_{L}$ presents inductive current, since its phase is late with respect to the tension of electric field to the angle $\frac{\pi}{2}$.

Maxwell equations for this case take the form

$$
\begin{aligned}
& \operatorname{rot} \vec{E}=-\mu_{0} \frac{\partial \vec{H}}{\partial t}, \\
& \operatorname{rot} \vec{H}=\vec{j}_{C}+\vec{j}_{L}=\varepsilon_{0} \frac{\partial \vec{E}}{\partial t}+\frac{1}{L_{k}} \int \vec{E} d t,
\end{aligned}
$$

where $\varepsilon_{0}$ and $\mu_{0}$ is dielectric and magnetic constant of vacuum, and the values $\vec{j}_{C}$ and $\vec{j}_{L}$ present respectively the bias current and conductivity. This form of writing of Maxwell equations taking into account the density of inductive current was for the first time proposed in work [7]. Further this question repeatedly was discussed in works [8-13].

From Eq. (2.7) we obtain

$$
\text { rot rot } \vec{H}+\mu_{0} \varepsilon_{0} \frac{\partial^{2} \vec{H}}{\partial t^{2}}+\frac{\mu_{0}}{L_{k}} \vec{H}=0
$$

For the case pour on, time-independent, equation (2.8) passes into the equation of London [14]

$$
\text { rot rot } \vec{H}+\frac{\mu_{0}}{L_{k}} \vec{H}=0
$$

where $\lambda_{L}^{2}=\frac{L_{k}}{\mu_{0}}$ is London depth of penetration.

It should be noted that in monograph [14] there is no equation (2.8), which characterizes the behavior of the nonmagnetized plasma in the variable fields, and equation for constant magnetic given only pour on (2.9).

Equation (2.7) indicates that neither dielectric nor magnetic of the permeability of the nonmagnetized plasma on frequency depend, but they are equal to the dielectric and magnetic constant of vacuum. Furthermore, this plasma characterizes one additional fundamental material parameter - specific kinetic inductance.

Equation (2.7) are accurate both for the constants and for the variables pour on. For the case of harmonics pour on $\vec{E}=\vec{E}_{0} \sin \omega t$ from (2.7) we obtain

$$
\text { rot } \vec{H}=\left(\varepsilon_{0} \omega-\frac{1}{L_{k} \omega}\right) \vec{E}_{0} \cos \omega t
$$

The value in the brackets is the reactive plasma conductivity $\sigma_{x}$, consequently

$$
\text { rot } \vec{H}=\sigma_{X} \vec{E}_{0} \cos \omega t
$$

where

$$
\sigma_{X}=\varepsilon_{0} \omega-\frac{1}{\omega L_{k}}=\varepsilon_{0} \omega\left(1-\frac{\omega_{\rho}^{2}}{\omega^{2}}\right)=\omega \varepsilon^{*}(\omega)
$$

Let us introduce the following designations

$$
\varepsilon^{*}(\omega)=\varepsilon_{0}\left(1-\frac{\omega_{\rho}^{2}}{\omega}\right)
$$

where $\omega_{\rho}^{2}=\frac{1}{\varepsilon_{0} L_{k}}$ is Langmuir frequency.

Then Eq. (2.17) can be rewritten

$$
\text { rot } \vec{H}=\omega \varepsilon_{0}\left(1-\frac{\omega_{\rho}^{2}}{\omega^{2}}\right) \vec{E}_{0} \cos \omega t
$$

or

$$
\text { rot } \vec{H}=\omega \varepsilon^{*}(\omega) \vec{E}_{0} \cos \omega t .
$$

In the literature in physics of plasma the value $\varepsilon^{*}(\omega)$ is accepted to call the dielectric constant of plasma $[1,2,3,4]$ depending on the frequency. Of this terminological and systematic error consists. In actuality the value $\varepsilon^{*}(\omega)$ includes simultaneously the dielectric constant of vacuum and the specific kinetic inductance of plasma and it is determined by the equation

$$
\varepsilon^{*}(\omega)=\frac{\sigma_{X}}{\omega}
$$

where $\sigma_{X}$ is the reactive plasma conductivity.

Is obvious that $\sigma_{X}$ can be recorded, also, on other

$$
\sigma_{X}=\varepsilon_{0} \omega-\frac{1}{\omega L_{k}}=\frac{1}{\omega L_{k}}\left(\frac{\omega^{2}}{\omega_{\rho}^{2}}-1\right)=\frac{1}{\omega L_{k} *},
$$

where

$$
L_{k} *(\omega)=\frac{L_{k}}{\left(\frac{\omega^{2}}{\omega_{\rho}^{2}}-1\right)}=\frac{1}{\sigma_{X} \omega} .
$$

Recorded thus value $L_{k} *(\omega)$ also includes and $\varepsilon_{0}$ and $L_{k}$ and by analogy from $\varepsilon^{*}(\omega)$ it can be named the inductance of plasma depending on the frequency.

The Eqs. (2.12) and (2.16) are equivalent and we with the identical success can assert that the plasma is characterized by the not frequency-dependent dielectric constant $\varepsilon^{*}(\omega)$, but by the frequency-dependent kinetic inductance $L_{k} *(\omega)$.

With the use of the parameters $\varepsilon^{*}(\omega)$ and $L_{k}^{*}(\omega)$ equation (2.10) it is possible to rewrite

$$
\operatorname{rot} \vec{H}=\omega \varepsilon^{*}(\omega) \vec{E}_{0} \cos \omega t
$$

or

$$
\text { rot } \vec{H}=\frac{1}{\omega L_{k} *(\omega)} \vec{E}_{0} \cos \omega t
$$

Equations (2.14) and (2.15) are also equivalent.

Thus, the parameter $\varepsilon^{*}(\omega)$ is not dielectric constant, although has its dimensionality. The same relates also to $L_{k}^{*}(\omega)$.

It is easy to see that

$$
\varepsilon^{*}(\omega)=\frac{\sigma_{X}}{\omega}, L_{k} *(\omega)=\frac{1}{\sigma_{X} \omega} .
$$


These equations determine physical sense and designation of the parameters $\varepsilon^{*}(\omega)$ and $L_{k} *(\omega)$.

To use $\varepsilon^{*}(\omega)$ and $L_{k}^{*}(\omega)$ for finding the energy according to the formulas

$$
W_{E}=\frac{1}{2} \varepsilon E_{0}^{2}
$$

and

$$
W_{j}=\frac{1}{2} L_{k} j_{0}^{2}
$$

is cannot. Work [15] gives the equation, which makes it possible to calculate the energy, accumulated in the nonmagnetized plasma, with the presence in it of variable electrical pour on with the use of the parameter of $\varepsilon^{*}(\omega)$

$$
W=\frac{1}{2} \cdot \frac{d\left[\omega \varepsilon^{*}(\omega)\right]}{d \omega} E_{0}^{2}
$$

However equation is given without what or substantiation, and is simple ugadano. Actually, if we in Eq. (2.16) substitute the value $\varepsilon^{*}(\omega)$, then we will obtain

$$
W_{\Sigma}=\frac{1}{2} \varepsilon_{0} E_{0}^{2}+\frac{1}{2} \cdot \frac{1}{\omega^{2} L_{k}} E_{0}^{2}=\frac{1}{2} \varepsilon_{0} E_{0}^{2}+\frac{1}{2} L_{k} j_{0}^{2} .
$$

This is the correct result, which indicates that the total energy, accumulated in the nonmagnetized plasma with the presence in it of variables pour on, is included energy of electrical pour on energy of kinetic electron motion. In work [15] this special feature of Eq. (2.16) is not examined

The same result is obtained, after using the equation

$$
W=\frac{1}{2} \cdot \frac{d\left[\frac{1}{\omega L_{k} *(\omega)}\right]}{d \omega} E_{0}^{2} .
$$

There are no of this equation in work [15] but the expression of energy, which of it follows, it coincides with Eq. (2.17). Thus, the authors of work [15] do not understand that role, which the kinetic inductance of charges in the formation of the energy, accumulated in the nonmagnetized plasma in the ac fields, plays.

\section{Transverse Plasma Resonance in that Nonmagnetized Plasma}

Let us show that under specific conditions in the nonmagnetized plasma can exist transverse the resonance with respect to the direction of propagation of electromagnetic waves.

Is known that the plasma resonance is longitudinal $[1,2,3,4]$. On existence in the limited nonmagnetized plasma of transverse resonance it communicates in work [16]. For explaining the conditions for the excitation of this resonance let us examine the long line, which consists of two ideally conducting planes, as shown in Figure 1. Let us examine the case, when this line is located in the vacuum.

If we to the extended line connect dc power supply $U$, then the energy, stored up in the electric field of line, will be written down

$$
W_{E \Sigma}=\frac{1}{2} \varepsilon_{0} E^{2} a b z=\frac{1}{2} C_{E \Sigma} U^{2},
$$

where $E=\frac{U}{a}$ is tension of electric field in the line.

The value

$$
C_{E \Sigma}=\varepsilon_{0} \frac{b z}{a}
$$

represents the total capacitance of the line, where $C_{E}=\varepsilon_{0} \frac{b}{a}$ linear of the capacity of line, and $\varepsilon_{0}$ is dielectric constant of vacuum.

Let us write down efficient potential electric field energy

$$
W_{E}=\frac{1}{2} \varepsilon_{0} E^{2}
$$

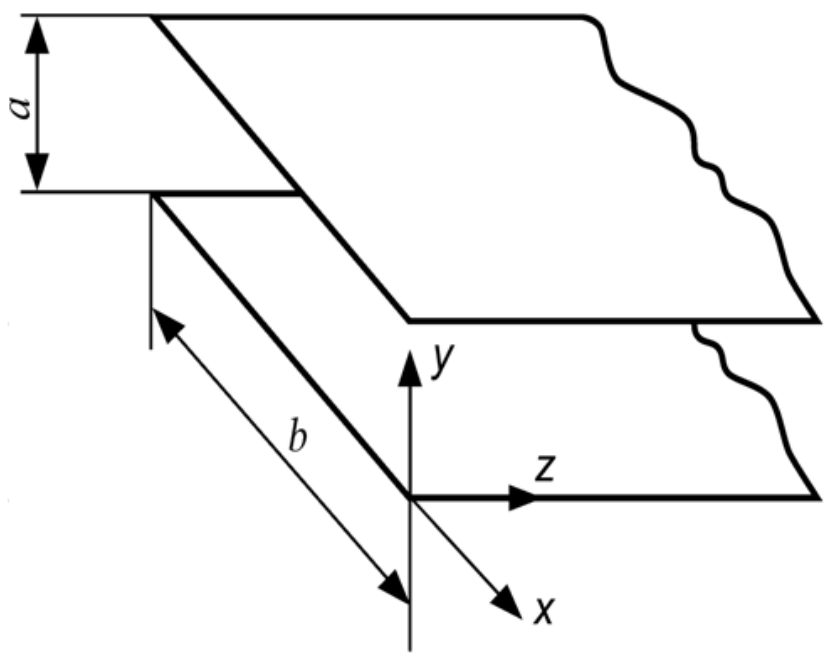

Figure 1. The two-wire circuit, which consists of two ideally conducting planes

If we line short out at a distance $\mathrm{z}$ and to connect to it the source of the direct current of $I$, then the energy, stored up in the magnetic field of line, will be written down

$$
W_{H \Sigma}=\frac{1}{2} \mu_{0} H^{2} a b z=\frac{1}{2} L_{H \Sigma} I^{2}
$$

since $H=\frac{I}{b}$, we will obtain

$$
L_{H \Sigma}=\mu_{0} \frac{a z}{b},
$$

where $L_{H \Sigma}$ is the summary inductance of line.

The value $L_{H}=\mu_{0} \frac{a}{b}$ is the running inductance of line, and $\mu_{\text {of } 0}$ corresponds to the specific inductance of medium, in this case of vacuum.

The specific energy of magnetic field will be written down

$$
W_{H}=\frac{1}{2} \mu_{0} H^{2}
$$

Subsequently for the larger clarity of the obtained results, together with their mathematical idea, we will use 
the method of equivalent diagrams. It is evident that with an increase in $\mathrm{z}, C_{E \Sigma}$ and $L_{H \Sigma}$ they increase, therefore the section of the line of the long $d z$ can be represented in the form the equivalent diagram, shown to Figure 2a.

If we in open-circuit line place the plasma, charge carriers in which can move without the losses, and pass through the flow line $I$, then charges they will stock kinetic energy. Since the transverse current density in this line is determined by the equation

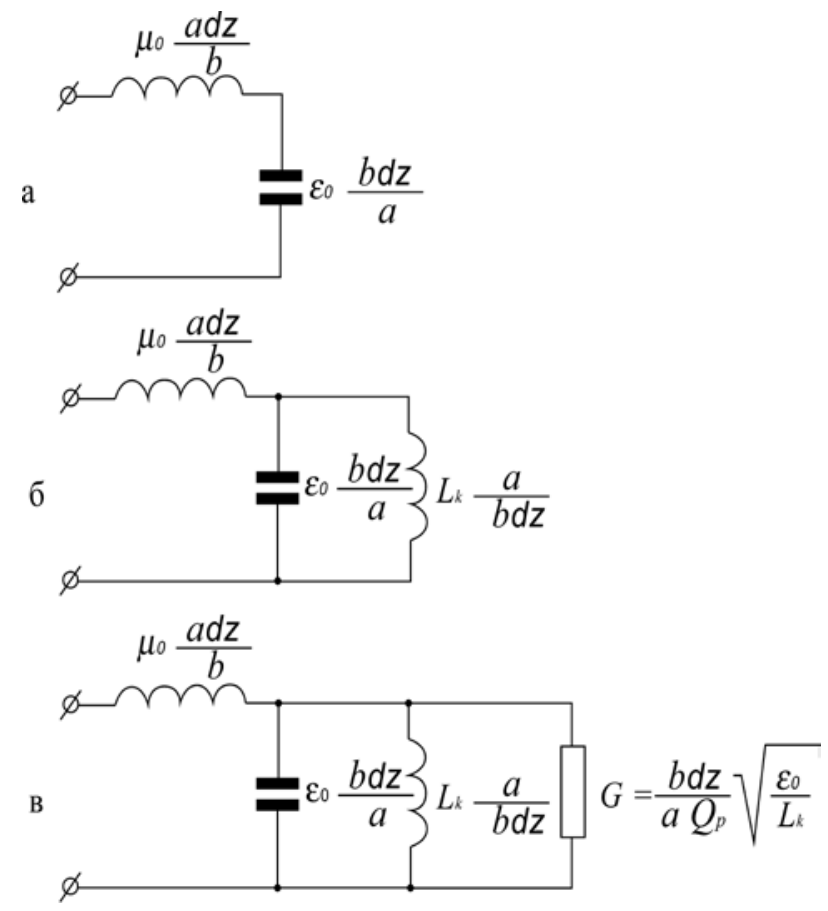

Figure 2. a - the equivalent the schematic of the section of the two-wire circuit; $\sigma$ - the equivalent the schematic of the section of the two-wire circuit, filled with plasma without the losses; в - the equivalent the schematic of the section of the two-wire circuit, filled by the dissipative plasma

$$
j=\frac{I}{b z}=n e V
$$

that summary kinetic energy of all moving charges will be written down

$$
W_{k \Sigma}=\frac{1}{2} \cdot \frac{m}{n e^{2}} a b z j^{2}=\frac{1}{2} \cdot \frac{m}{n e^{2}} \frac{a}{b z} I^{2}
$$

Is fulfilled also the equation

$$
W_{k \Sigma}=\frac{1}{2} L_{k \Sigma} I^{2}
$$

where $L_{k \Sigma}$ - complete kinetic inductance of line. Consequently

$$
L_{k \Sigma}=\frac{m}{n e^{2}} \cdot \frac{a}{b z}
$$

Thus, the value

$$
L_{k}=\frac{m}{n e^{2}}
$$

the role of the specific kinetic inductance of this medium plays.
We already previously introduced this value in another manner (see Eq. (2.4)).

Equation (3.2) is obtained for the case of the direct current, when current distribution is uniform.

From Eq. (3.1) is evident that in contrast to and of the value of with an increase in does not increase, but it decreases. Connected this with the fact that with an increase in a quantity of parallel-connected inductive elements grows. The equivalent the schematic of the section of the line, filled with the plasma, in which there are no losses, it is shown in Figure 2b. Line itself in this case will be equivalent to parallel circuit with the lumped parameters $C=\frac{\varepsilon_{0} b z}{a}$ and $L=\frac{L_{k} a}{b z}$.

But if we calculate the resonance frequency of this outline, then it will seem that this frequency generally not on what sizes depends, actually

$$
\omega_{\rho}^{2}=\frac{1}{C L}=\frac{1}{\varepsilon_{0} L_{k}}=\frac{n e^{2}}{\varepsilon_{0} m}
$$

Is obtained the very interesting result, which speaks, that the resonance frequency macroscopic of the resonator examined does not depend on its sizes. Impression can be created, that this is plasma resonance, since. The obtained value of resonance frequency exactly corresponds to the value of this resonance. But it is known that the plasma resonance characterizes longitudinal waves in the long line they, while occur transverse waves. In the case examined the value of the phase speed in the direction of is equal to infinity and the wave vector $\vec{k}_{z}=0$. This result corresponds to the solution of system of equations (2.7) for the line with the assigned configuration (Figure 1). From Eqs. (2.8) known result [1,2,3,4]. In this case the wave number is determined by the equation

$$
k_{z}^{2}=\frac{\omega^{2}}{c^{2}}\left(1-\frac{\omega_{\rho}^{2}}{\omega^{2}}\right)
$$

and the group and phase speeds

$$
\begin{gathered}
v_{g}^{2}=c^{2}\left(1-\frac{\omega_{\rho}^{2}}{\omega^{2}}\right) \\
v_{p}^{2}=\frac{c^{2}}{\left(1-\frac{\omega_{\rho}^{2}}{\omega^{2}}\right)}
\end{gathered}
$$

where $c=\left(\frac{1}{\mu_{0} \varepsilon_{0}}\right)^{1 / 2}$ is speed of light in the vacuum.

In this plasma the phase speed of electromagnetic wave is equal to infinity. Consequently, at each moment of time pour on distribution and currents in this line uniform and it does not depend on the coordinate, but current in the planes of line in the direction of is absent. Consequently, current in the planes of line in the direction $\mathrm{z}$ is absent. This, from one side, it means that the inductance of will not have effects on electrodynamic processes in this line, but instead of the conducting planes can be used any 
planes or devices, which limit plasma on top and from below.

From Eqs. (3.3), (3.4) and (3.5) is evident that at the point of $\omega=\omega \rho$ occurs the transverse resonance with the infinite quality. The fact that in contrast to the plasma, this resonance is transverse, will be one can see well for the case, when the quality of this resonance does not be equal to infinity. In this case $k_{z} \neq 0$ and in the line will be extended the transverse wave, the direction of propagation of which will be perpendicular to the direction of the motion of charges. The examination of this task was begun from the examination of the plasma, limited from two sides by the planes of long line. But in the process of this examination it is possible to draw the conclusion that the frequency of this resonance generally on the dimensions of line does not depend. It means, resonance will be observed, also, in the unbounded medium. Thus, in the limitless plasma besides the Langmuir resonance, which characterizes longitudinal waves, can exist transverse resonance. Since the frequencies of these resonances coincide, they are degenerate. It should be noted that the fact of existence of this resonance previously was not realized and in the literature it was not described.

Let us examine energy processes, the occurring in the plasma in the case of absence losses.

Pour on the characteristic impedance of plasma, which gives the relation of the transverse components of electrical and magnetic, let us determine from the equation

$$
Z=\frac{E_{y}}{H_{x}}=\frac{\mu_{0} \omega}{k_{z}}=Z_{0}\left(1-\frac{\omega_{\rho}^{2}}{\omega^{2}}\right)^{-1 / 2}
$$

where $Z_{0}=\sqrt{\frac{\mu_{0}}{\varepsilon_{0}}}$ is characteristic (wave) resistance of vacuum.

The obtained value of $\mathrm{Z}$ is characteristic for the transverse electrical waves in the waveguides. It is evident that when $\omega \rightarrow \omega_{\rho}, Z \rightarrow \infty$, and $H_{x} \rightarrow 0$. When $\omega>\omega_{\rho}$ in the plasma there is electrical and magnetic component of field. The specific energy of these fields on it will be written down

$$
W_{E, H}=\frac{1}{2} \varepsilon_{0} E_{0 y}^{2}+\frac{1}{2} \mu_{0} H_{0 x}^{2} .
$$

Thus, the energy, concluded in the magnetic field, in $\left(1-\frac{\omega_{\rho}^{2}}{\omega^{2}}\right)$ of times is less than the energy, concluded in the electric field. Let us note that this examination, which is traditional in the electrodynamics, is not complete, since in this case is not taken into account one additional form of energy, namely kinetic energy of charge carriers. Occurs that pour on besides the waves of electrical and magnetic, that carry electrical and magnetic energy, in the plasma there exists even and the electrickinetic wave, which carries kinetic energy of current carriers. The specific energy of this wave is written

$$
W_{k}=\frac{1}{2} L_{k} j_{0}^{2}=\frac{1}{2} \cdot \frac{1}{\omega^{2} L_{k}} E_{0}^{2}=\frac{1}{2} \varepsilon_{0} \frac{\omega_{\rho}^{2}}{\omega^{2}} E_{0}^{2}
$$

Thus, total specific energy is written as

$$
W_{E, H, j}=\frac{1}{2} \varepsilon_{0} E_{0 y}^{2}+\frac{1}{2} \mu_{0} H_{0 x}^{2}+\frac{1}{2} L_{k} j_{0}^{2}
$$

Thus, for finding the total energy, by the prisoner per unit of volume of plasma, calculation only fields $E$ and $H$ it is insufficient.

At the point $\omega=\omega_{\rho}$

$$
W_{H}=0, W_{E}=W_{k},
$$

i.e. magnetic field in the plasma is absent, and plasma presents macroscopic electromechanical resonator with the infinite quality, wpresounding at the frequency.

Since with the frequencies $\omega>\omega_{\rho}$ the wave, which is extended in the plasma, it bears on itself three forms of the energy: electrical, magnetic and kinetic, then this wave can be named electromagnetic kinetic wave. Kinetic wave represents the wave of the current density of $\vec{j}=\frac{1}{L_{k}} \int \vec{E} d t$. This wave is moved with respect to the electrical wave the angle $\pi / 2$.

If losses are located, moreover completely it does not have value, by what physical processes such losses are caused, then the quality of plasma resonator will be finite quantity. For this case of Maxwell equation they will take the form

$$
\begin{aligned}
& \operatorname{rot} \vec{E}=-\mu_{0} \frac{\partial \vec{H}}{\partial t}, \\
& \operatorname{rot} \vec{H}=\sigma_{p . e f} \vec{E}+\varepsilon_{0} \frac{\partial \vec{E}}{\partial t}+\frac{1}{L_{k}} \int \vec{E} d t .
\end{aligned}
$$

The presence of losses is considered by the term $\sigma_{\text {p.ef }} \vec{E}$,, and, using near the conductivity of the index ef, it is thus emphasized that us does not interest very mechanism of losses, but only very fact of their existence interests. Let us examine the method of measuring $\sigma_{\text {p.ef }}$. For measuring should be selected the section of line by the length, whose value is considerably lower than the wavelength in the plasma. This section will be equivalent to outline with the lumped parameters

$$
\begin{gathered}
C=\varepsilon_{0} \frac{b z_{0}}{a}, \\
L=L_{k} \frac{a}{b z_{0}}, \\
G=\sigma_{\rho . e f} \frac{b z_{0}}{a},
\end{gathered}
$$

where o $G$ is conductivity, connected in parallel $C$ and $L$.

The conductivity $G$ of this outline is determined by the equation

$$
G=\frac{1}{Q_{\rho}} \sqrt{\frac{C}{L}}
$$

where $Q_{\rho}$ is quality of the resonator.

From where, taking into account (3.8 - 3.10), we obtain 


$$
\sigma_{\rho . e f}=\frac{1}{Q_{\rho}} \sqrt{\frac{\varepsilon_{0}}{L_{k}}}
$$

consequently, measuring its own quality of plasma resonator, it is possible to determine $\sigma_{\text {p.ef }}$.

Using Eqs. (3.6) and (3.10) we will obtain

$$
\begin{aligned}
& \operatorname{rot} \vec{E}=-\mu_{0} \frac{\partial \vec{H}}{\partial t}, \\
& \operatorname{rot} \vec{H}=\frac{1}{Q_{\rho}} \sqrt{\frac{\varepsilon_{0}}{L_{k}}} \vec{E}+\varepsilon_{0} \frac{\partial \vec{E}}{\partial t}+\frac{1}{L_{k}} \int \vec{E} d t .
\end{aligned}
$$

The equivalent the schematic of this line, filled with dissipative plasma, is represented in Fig 2B.

Let us examine the solution of system of equations (3.11) at the point of, in this case, since

$$
\varepsilon_{0} \frac{\partial \vec{E}}{\partial t}+\frac{1}{L_{k}} \int \vec{E} d t=0
$$

we obtain

$$
\begin{aligned}
& \text { rot } \vec{E}=-\mu_{0} \frac{\partial \vec{H}}{\partial t}, \\
& \text { rot } \vec{H}=\frac{1}{Q_{P}} \sqrt{\frac{\varepsilon_{0}}{L_{k}}} \vec{E} .
\end{aligned}
$$

The solution of this system of equations is well known [5]. If there is an interface between the vacuum and the medium, whose parameters are determined by equations (3.12), then the surface impedance of medium is written

$$
Z=\frac{E_{t g}}{H_{t g}}=\sqrt{\frac{\omega_{p} \mu_{0}}{2 \sigma_{p . e f .}}}(1+i)
$$

where $\sigma_{p . e f}=\frac{1}{Q_{p}} \sqrt{\frac{\varepsilon_{0}}{L_{k}}}$.

The wave, which exits into the depths of the medium, diminishes according to the law $e^{-\frac{z}{\delta_{e f}}} \cdot e^{-i \frac{z}{\delta_{e f}}}$, phase speed in this case it is determined by the equation of

$$
v_{p}=\omega \sigma_{p . e f}
$$

where of $\delta_{p . e f}^{2}=\frac{2}{\mu_{0} \omega_{p} \sigma_{p . e f}}$ - effective depth of penetration of field into the plasma. The obtained equations characterize wave process in the plasma. For good conductors of $\frac{\sigma_{e f}}{\omega \varepsilon_{0}}>>1$ and then wavelength in this medium of

$$
\lambda_{\mathrm{g}}=2 \pi \delta
$$

is considerably less than wavelength in the free space. Subsequently of us it will interest the case, when at the point $\omega=\omega_{\mathrm{p}} \lambda_{\mathrm{g}}>>\lambda_{0},\left.v_{p}\right|_{\omega=\omega \mathrm{p}}>>\mathrm{c}$.

The examination of the electrodynamic properties of plasma is carried out nontraditionally. We not at all introduced this concept as the frequency dependent dielectric constant of plasma. This made possible to understand, that $\varepsilon^{*}(\omega)$ is purely mathematical, but not physical concept, and that the dielectric constant of plasma on frequency does not depend, but is equal to the dielectric constant of vacuum. From other side, this approach gave to us the possibility to understand, what energy processes occur in the plasma, and what forms of energy in it are stocked and are extended.

\section{Practical Results}

Since with the aid of the plasma can be created macroscopic single-frequency resonator, this resonator can be used for development and creating the new class of electrokinetic lasers. This resonator can be also used as band-pass filter.

With the sufficiently great significances of $Q_{p}$ energy of magnetic fields on at the resonance frequency considerably less than kinetic energy of the current carriers and energy of electrostatic pour on. Furthermore, the phase speed in this resonator under specific conditions can be considerably more than the speed of light. Therefore, with the excitation of transverse plasma resonance it is possible to place

$$
\begin{aligned}
& \text { rot } \vec{E} \cong 0, \\
& \frac{1}{Q_{p}} \sqrt{\frac{\varepsilon_{0}}{L_{k}}} \vec{E}+\varepsilon_{0} \frac{\partial \vec{E}}{\partial t}+\frac{1}{L_{k}} \int \vec{E} d t=\vec{j}_{C T},
\end{aligned}
$$

where $\vec{j}_{C T}$ is density of strange currents.

Differentiating (4.1) on the time and after dividing into $\varepsilon_{0}$ we will obtain

$$
\omega_{p}^{2} \vec{E}+\frac{\omega_{p}}{Q_{p}} \cdot \frac{\partial \vec{E}}{\partial t}+\frac{\partial^{2} \vec{E}}{\partial t^{2}}=\frac{1}{\varepsilon_{0}} \cdot \frac{\partial \vec{j}_{s d}}{\partial t} .
$$

If we (4.2) integrate over the surface of normal to the vector $\vec{E}$ and to designate

$$
\Phi_{E}=\int \vec{E} d \vec{S}
$$

where $\Phi_{E}$ is the electric flux.

And further we will obtain

$$
\omega_{p}^{2} \Phi_{E}+\frac{\omega_{p}}{Q_{p}} \cdot \frac{\partial \Phi_{E}}{\partial t}+\frac{\partial^{2} \Phi_{E}}{\partial t^{2}}=\frac{1}{\varepsilon_{0}} \cdot \frac{\partial I_{s d}}{\partial t}
$$

where $I_{s d}$ is strange current.

Equation (4.3) is the equation of harmonic oscillator with the right side, characteristic for the two-level laser [17]. If the source of excitation is absent, then we deal concerning "cold" laser resonator, in which the fluctuations attenuate exponentially

$$
\Phi_{E}(t)=\Phi_{E}(0) e^{i \omega_{P} t} \cdot e^{-\frac{\omega_{P}}{2 Q_{P}} t}
$$

i.e. the macroscopic electric flux of will oscillate with the frequency, relaxation time in this case is determined by the equation

$$
\tau=\frac{2 Q_{P}}{\omega_{P}}
$$


If resonator is excited by strange currents, then this resonator presents band-pass filter with the resonance frequency to equal plasma frequency and the pass band

$$
\Delta \omega=\frac{\omega_{p}}{2 \Theta_{p}} .
$$

Another important practical application of transverse plasma resonance is possibility its use for warming-up and diagnostics of plasma. If the quality of this resonator is great, which can be achieved at moderate concentrations of plasma and its high temperature, then in this resonator can be obtained the high levels of electrical pour on, and it means high energies of charge carriers.

Plasma the resonator examined is not difficult to coordinate with the line of communications. The equivalent resistance of resonator at the point $\omega=\omega_{p}$ will be written down

$$
R=\frac{1}{G}=\frac{a Q_{P}}{b z} \sqrt{\frac{L_{k}}{\varepsilon_{0}}} .
$$

if $b=b_{L}$, then matching condition they take the form

$$
\begin{aligned}
& \frac{a_{L}}{b_{L}} \sqrt{\frac{\mu_{0}}{\varepsilon_{0}}}=\frac{a Q_{p}}{b z_{0}} \sqrt{\frac{L_{k}}{\varepsilon_{0}}}, \\
& \frac{a \Theta_{p}}{a_{L} z_{0}} \sqrt{\frac{L_{k}}{\mu_{0}}}=1
\end{aligned}
$$

This one should remember that selected the length of the resonator $\mathrm{z}_{0}$ should be from the condition $\mathrm{z}_{0}<<\left.\lambda_{\mathrm{g}}\right|_{\omega=}$ $\omega_{\mathrm{p}}$.

With the creation on the basis of the plasma resonator of different devices can arise the need for the agreement of this resonator with the free space. It is obvious that in this case must be observed the condition

$$
\sqrt{\frac{\mu_{0}}{\varepsilon_{0}}}=\frac{a Q_{p}}{b z_{0}} \sqrt{\frac{L_{k}}{\varepsilon_{0}}}
$$

or

$$
\frac{a Q_{p}}{b z_{0}} \sqrt{\frac{L_{k}}{\mu_{0}}}=1
$$

Transverse plasma resonance can be used also for the warming-up of plasma and its diagnostics. The effectiveness of warming-up is defined by the fact that the losses in the high-temperature plasma are small, and, therefore, can be achieved the high quality of resonator and as consequence the high values of electrical pour on in the Ger. The resonance indicated can be used for purposes of diagnostics of plasma for density measurement of free charge carriers.

\section{Conclusion}

It is shown that in the nonmagnetized plasma, besides longitudinal Langmuir resonance, with satisfaction of the specified boundary conditions, can exist the transverse plasma resonance. It is known that with the nuclear explosions the electromagnetic radiation in the very wide frequency band is observed, up to the radio-frequency range. And if the emission in field of light range can be explained by the emission of separate atoms, then emission in the region of radio-frequency band can be caused only by collective processes, which occur in the confined plasma. The use of transverse resonance makes it possible to create resonators and band-pass filters, and also lasers on the collective plasma oscillations. Transverse plasma resonance can be used also for the warming-up of plasma and its diagnostics. Is introduced the concept of magnetoelectrokinetic waves.

\section{References}

[1] V. L. Ginzburg, The propagation of electromagnetic waves in a plasma.-M.: Science. 1967.

[2] A. I. Akhiezer, Plasma Physics Nauka, Moscow, 1974.

[3] A. F. Aleksandrov, L.S. Bogdankevich, A. A. Rukhdze, Oscillations and waves in plasma media, Moscow University Publishers, 1990.

[4] A. A. Artsimovich, R. Z. Sagdeev, Plasma Physics for Physicists. M: Atomizdat, 1979.

[5] F.F. Mende, A.I. Spitsyn, Surface impedance in superconductors, Kiev, Naukova Dumka, 1985.

[6] F. F. Mende, Role and place of the kinetic inductance of charges in classical electrodynamics, Engineering Physics, № 11, 2012.

[7] F. F. Mende, On refinement of equations of electromagnetic induction, Kharkov, deposited in VINITI, No 774-B88 Dep., 1988

[8] F. F. Mende, Are there errors in modern physics. Kharkov, Constant, 2003.

[9] F.F. Mende, On refinement of certain laws of classical electrodynamics,arXiv.org/abs/physics/0402084.

[10] F. F. Mende, Consistent electrodynamics, Kharkov NTMT, 2008.

[11] F. F. Mende, Consistent electrodynamics and the threat of nuclear Space terrorism. Kharkov NTMT, 2008.

[12] F. F. Mende, Great misconceptions and errors physicists XIX-XX centuries. Revolution in modern physics, Kharkiv NTMT, 2010.

[13] F. F. Mende New electrodynamics, Revolution in modern physics. Kharkov NTMT, 201.

[14] F. London, Superfluids. Vol.1. Microscopic theory of superconductivity.-Nev York: Dower publ., 1950.

[15] L. D. Landau, E.M. Lifshits, Electrodynamics of continuous media. Moscow 1973.

[16] F. F. Mende, Transversal plasma resonance in a nonmagnetized plasma and possibilities of practical employment of it, arXiv.org/abs/physics/0506081.

[17] A.Yriv, Quantum electrodynamics and nonlinear optics. Sov. Radio: Moscow 1973. 